LA-UR-01-3364

Approved for public release;

distribution is unlimited.

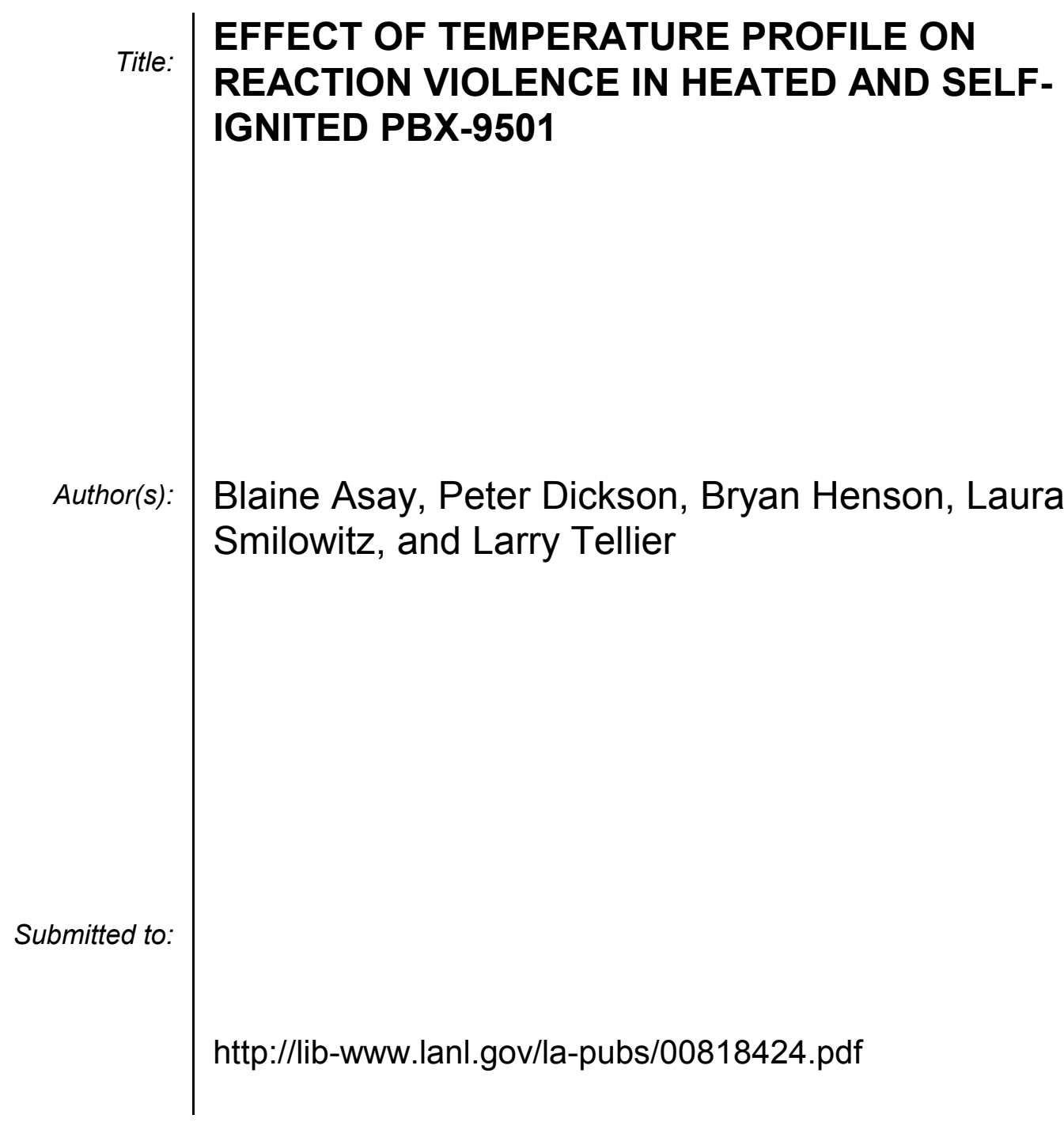

Los Alamos National Laboratory, an affirmative action/equal opportunity employer, is operated by the University of California for the U.S. Department of Energy under contract W-7405-ENG-36. By acceptance of this article, the publisher recognizes that the U.S. Government retains a nonexclusive, royaltyfree license to publish or reproduce the published form of this contribution, or to allow others to do so, for U.S. Government purposes. Los Alamos National Laboratory requests that the publisher identify this article as work performed under the auspices of the U.S. Department of Energy. Los Alamos National Laboratory strongly supports academic freedom and a researcher's right to publish; as an institution, however, the Laboratory does not endorse the viewpoint of a publication or guarantee its technical correctness. 


\title{
EFFECT OF TEMPERATURE PROFILE ON REACTION VIOLENCE IN HEATED AND SELF-IGNITED PBX-9501
}

\author{
Blaine Asay, Peter Dickson, Bryan Henson, \\ Laura Smilowitz, and Larry Tellier
}

Los Alamos National Laboratory, Los Alamos, NM 87545

\begin{abstract}
Historically, the location of ignition in heated explosives has been implicated in the violence of subsequent reactions. This is based on the observation that typically, when an explosive is heated quickly, ignition occurs at the surface, leading to premature failure of confinement, a precipitous drop in pressure, and failure of the reaction. During slow heating, reaction usually occurs near the center of the charge, and more violent reactions are observed. Many safety protocols use these global results in determining safety envelopes and procedures. We are conducting instrumented experiments with cylindrical symmetry and precise thermal boundary conditions which are beginning to show that the temperature profile in the explosive, along with the time spent at critical temperatures, and not the location of ignition, are responsible for the level of violence observed. Microwave interferometry was used to measure case expansion velocities which can be considered a measure of reaction violence. We are using the data in a companion study to develop better kinetic models for HMX and PBX 9501. Additionally, the spatiallyand temporally-resolved temperature data are being made available for those who would like to use them.
\end{abstract}

\section{INTRODUCTION}

Thermal studies of explosives have traditionally measured the time to ignition as a function of an isothermal temperature as the figure of merit. Kinetics for the process have been derived for these measurements. For example, the one-dimensional time to explosion experiment (ODTX) has been used for a number of years, and has produced the best results to date [1]. In this experiment, a sphere of explosive is placed between two heated anvils and the time required for significant reaction is recorded. The results are then plotted as $1 / \mathrm{T}$ vs. time. The location of the ignition is not measured and is not known.

Over many years of slow and fast heating tests, the conclusion has been drawn that the location of ignition governs the violence of the explosive response. A slow heat scenario will allow the center of the HE to heat up sufficiently so that once self- heating begins, the reaction runs away at the center. A rapid heating produces high temperatures first at a region near the boundary, and runaway thus occurs there. It has been noted that fast cookoff usually results in a less violent reaction than does slow cookoff, and since the location of ignition is so different between the two cases, this has been thought of as being paramount.

We have conducted a series of tests wherein we measure a temporally and spatially resolved thermal profile [2]. This allows us to ascertain not only at what time the reaction begins, but the location as well. Using these two measures, we have been able to modify the classic ODTX kinetics as well as investigate the effects of location of ignition on violence, separate from the heating rate. This study was designed to investigate the role of thermally 
induced damage on the violence as measured by wall velocity.

\section{EXPERIMENTAL}

The experiments have been previously described [Peter Dickson, 1999 \#2] but have been slightly modified for the current study. We have used PBX 9501 which is 95\% HMX and 5\% binder. The explosive is encased in 2 copper half-cylinders split down the middle (each $10.3 \mathrm{~mm}$ in length $\mathrm{x} 19.4$ diameter, with $3.7 \mathrm{~mm}$-thick wall). Heat is supplied to heating wire wrapped around the $\mathrm{Cu}$ cylinders via two circuits, one on the top and one on the bottom, each individually controlled. Five thermocouples are placed at the union of the two cylinders at different radial positions (see Fig. 1). The experiment is designed so that the centerline will be the hottest point with heat flow occurring out of each end cap. The system is held together by threaded rod, but is not sealed against gas loss.

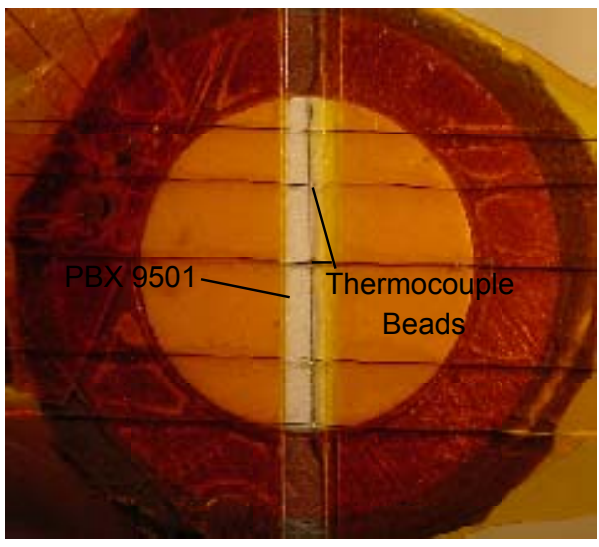

FIGURE 1. Photograph of one-half of experiment showing copper cylinder, explosive and thermocouples

We measure the wall velocity using a $35 \mathrm{GHz}$ microwave interferometer $(\lambda=8.45 \mathrm{~mm})$. The interferometer is coupled to a PTFE waveguide that is inserted into the armored box containing the explosive. There is a small disk attached to the wall of the experiment that reflects the microwaves (see Fig. 2). We have also reflected the microwaves from the wall of the cylinder with good results. The quadrature signal is then analyzed and position as a function of time is extracted. The data are differentiated to obtain velocities.

For this set of experiments we applied a steady heating ramp until the temperature reached $185 \mathrm{C}$. This is slightly above the phase transition temperature, but below the temperature at which rapid reaction begins. This was then maintained for a predetermined amount of time after which a second ramp was imposed, and self-ignition resulted. The experiments were designed to provide a varying amount of time during which thermal damage could occur. The second ramp accelerated the self-ignition time for convenience.

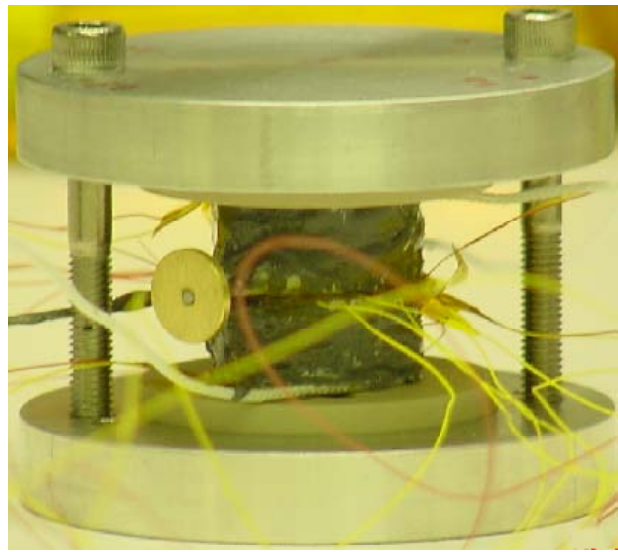

FIGURE 2. Photograph of assembled experiment showing optional microwave reflector.

\section{RESULTS}

Soak times of $1,2,3,4,6$, and 8 hours were used, with two experiments performed using 1 hour soak times to examine reproducibility. For presentation here, the time at which the second ramp was started for each experiment was shifted so that each experiment had a common point. This was done to compare self-ignition times. Figure 3 shows the entire temperature histories while Fig. 4 shows an enlarged view of the ignition region.

The thermocouple records shown are from the regions of the charge that showed the fastest temperature rise. Although each experiment had uniform heating that was well controlled, they did not all ignite at the same radial position. 


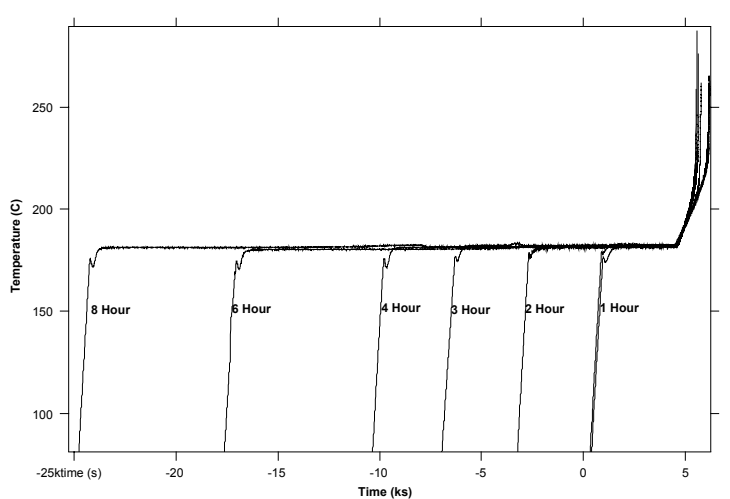

FIGURE 3. Temperature traces showing effect of soak time on self-ignition time. Times shifted for comparison.

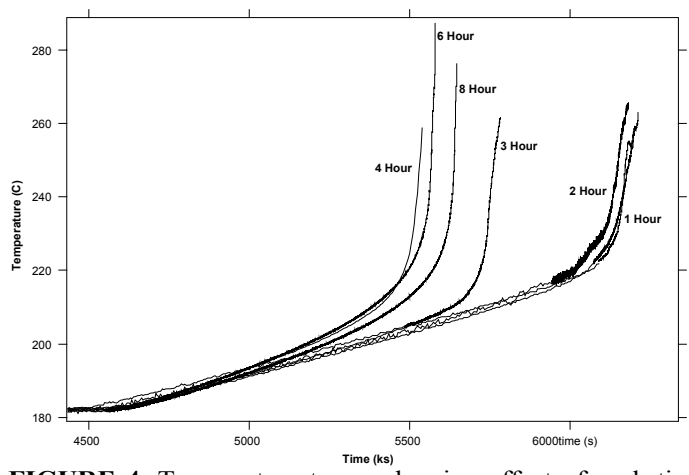

FIGURE 4. Temperature traces showing effect of soak time on self-ignition time. Times shifted for comparison. Time scale enlarged for clarity.

The experiments with the 1 hour soak showed good reproducibility. The successive shots followed a predictable pattern up to and including the 4-hour soak, with each one igniting at earlier times (see Fig. 4). This was expected because with increasing soak time, more damage (e.g., thermal decomposition and fracture) is occurring and the chemical reactions are further advanced. However, the experiment with the 6-hour soak showed a slightly increased ignition time and the test with the 8-hour soak showed a much longer time to ignition. This most likely results from an increasingly significant loss of gaseous products from the system. As heating time increases, porosity of the explosive sample also increases. At some point, the porosity will become interconnected, permitting wholesale loss of products. Because the major exothermic reactions occur in the gas phase, loss of these products results in an increase in ignition time. It is widely known that gas loss can have a profound effect on reaction times. We have maintained however that for relatively short experiments, or very large ones, that the permeation of the gas out of the system is slow enough so as not to affect the outcome. Permeabilities of pressed unreacted systems are very low. This set of experiments demonstrates that fact.

We have observed in each experiment that near the end of the exponentional temperature rise there is an inflection point in the temperature record. This could be the signature of the melt, or another, unidentified chemical step. We took the derivative of temperature with respect to time at that point. Those results are presented in Fig. 5 along with the measurement of the self-ignition time. The derivative results are linear with heating time. This could illustrate that while globally the time to ignition decreases and then increases because of gas loss, the chemistry occurring at the point of maximum heat release maintains a memory of the thermal history. This measurement could provide a quantitative indicator of reaction violence in that it demonstrates a large increase in heat release (at least three-fold in this example) as a function of damage state.

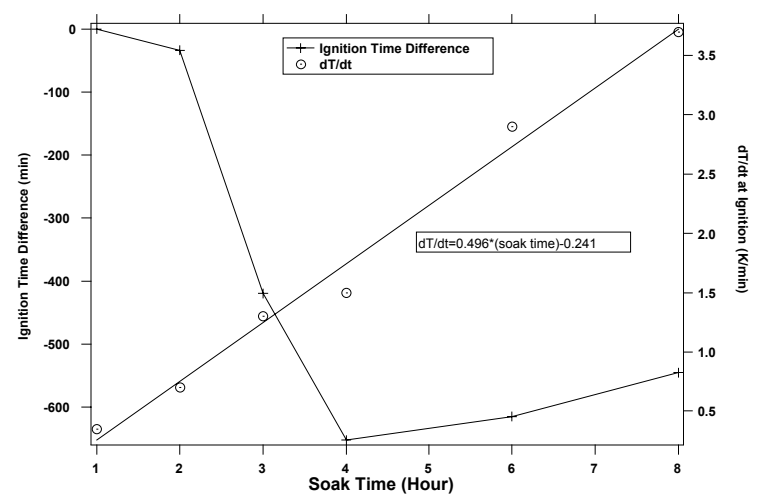

FIGURE 5. Plot showing the self-ignition time difference as a function of soak time and the derivative of the temperature at the endotherm occurring at runaway.

The experiments have been modeled using the 4step kinetics reported earlier [Peter Dickson, 1999 \#2]. The parameters were chosen so as to provide a best fit for the 1-hour case, and then held constant for the remaining cases. The results are presented in Fig. 6. The overall agreement is good across the entire spectrum, but there are some differences. The rise in the calculated baseline temperature for the case with the longest soak time is consistent with the notion that there is appreciable gas loss for this particular experiment. The code predicts heating that is not present in the data. The source of this heating would arise from gas phase reactions. 


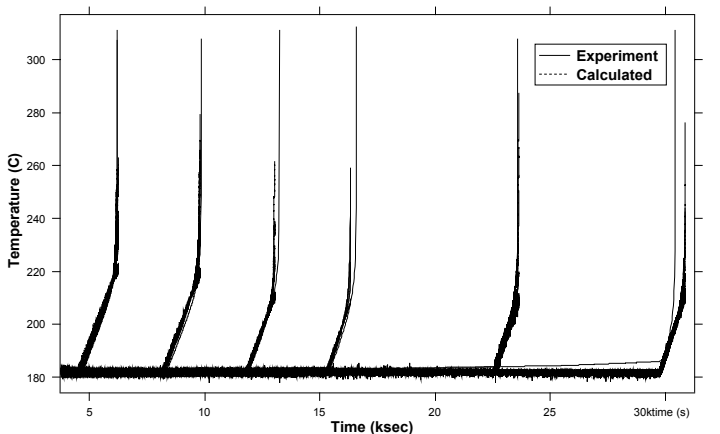

FIGURE 6. Comparison of model prediction with experimental values.

The case expansion velocities were measured using a microwave interferometer. We performed one experiment wherein we purposely detonated the encased explosive. The measured wall velocity was approximately $500 \mathrm{~m} / \mathrm{s}$, which compares favorably to a calculation using the Gurney method. The velocities for each of the cookoff experiments have been tabulated and in every case the values have been approximately $100-150 \mathrm{~m} / \mathrm{s}$, well below that of a detonation. No distinguishing differences in velocity were noted for the experiments performed under different thermal conditions. One interpretation of this result is that there was no marked difference in reaction violence with the large changes in damage state. However, the difference in reaction rate measured at late times (see Fig. 5) demonstrates that there is a significant difference in the HE behavior at during ignition. The case expansion velocity is an integrated measurement of reaction history, and as such, may not be a reliable indicator of reaction violence in this geometry or at this scale.

Fragments were collected after each experiment. Their size and distribution corroborated the velocity measurements, in that no evidence of detonation was found, and no major differences in fragment distribution with experimental change were noted. However, further analysis is required.

\section{DISCUSSION AND CONCLUSIONS}

These experiments were designed to examine the violence of reaction when the explosive was subjected to different degrees of thermal damage.
We found that, with the prescribed heating profiles, no differences in violence were obtained. We also noted that the location of ignition varied between the experiments from near the case to the center of the charge. Thus, we have shown that the ignition location is not a primary factor in the determination of violence for a given reaction. The experiments all had different soak times, so the thermal conditions were somewhat different. However, we do not believe that this changes this basic conclusion.

We believe that the second temperature ramp may have masked subtle reactive behavior, in that it overdrove ignition somewhat. Our next series of experiments are designed to modify this part of the profile in an effort to drive the reaction to different levels of violence.

We have also shown that the definition of reaction violence needs to be clarified, in that two separate measures provided different conclusions. We need to better understand the differences in measured reaction rates as a function of damage state, and the reasons that these differences were not reflected in case expansion velocities.

The thermal profiles from these experiments are available in text format to facilitate comparison with various computer models. They can be secured from the authors.

\section{ACKNOWLEDGEMENTS}

We acknowledge the support of the LANL HE Science and Surety programs that made this work possible.

\section{REFERENCES}

1. McGuire, R.R. and C.M. Tarver. in Seventh Symposium (International) on Detonation. 1981. Annapolis, MD.

2. Peter Dickson, et al. Measurement of Phase Change and Thermal Decomposition Kinetics During cookoff of PBX 9501. in Shock Compression of Condensed Matter, American Physical Society Topical Conference. 1999. Snowbird, UT. 